\title{
Improvement in Protracted Wound Healing by Topical Cream Containing Lipopolysaccharide Derived from Pantoea agglomerans
}

\author{
ATSUTOMO MORISHIMA ${ }^{1}$ and HIROYUKI INAGAWA ${ }^{2}$ \\ ${ }^{1}$ Lavita Medical Clinic, Osaka, Japan; \\ ${ }^{2}$ Faculty of Medicine, Kagawa University, Kagawa, Japan
}

\begin{abstract}
Background/Aim: The wound-healing effect of lipopolysaccharide (LPS) reportedly results from its ability to induce the removal of foreign bodies, anti-inflammatory function, and tissue-repair function. We reported the improvement of patients with protracted wound healing after the dermal administration of topical cream containing LPS derived from Pantoea agglomerans, which is a symbiotic bacterium present in wheat and confirmed as safe. Patients and Methods: Topical cream with LPS was applied on four patients who showed protracted wound healing, after their informed consent. The wound was evaluated on the basis of the change in the approximate wound volume calculated from the width, length, and depth of the wound. Results: Case 1: A 76-year-old man developed infection at the puncture site after endovascular laser treatment of the right lower extremity varicose veins and suffered protracted wound healing. The wound was treated with gentamicin application containing LPS, and shrank in two weeks. Case 2: A 72-year-old man developed a wound infection and had purulent drainage one week after endovascular laser treatment of the left lower extremity varicose veins. The wound was closed in one month using gentamicin application containing LPS. Case 3: A 67-year-old woman with protracted wound healing developed infection in the right inguinal region after the surgical treatment of acute aortic dissection and experienced complete wound dehiscence. The wound shrank one week after gauze packing with LPS and was temporarily sutured. The wound was completely closed in two weeks with gentamicin application containing LPS.
\end{abstract}

Correspondence to: Dr. Atsutomo Morishima, Medical director, Lavita Medical Clinic, Hiradai-cho, Moriguchi-shi, Osaka, 570-0078, Japan. Tel/Fax: +81 669911000, e-mail: atsu5426@yahoo.co.jp

Key Words: Protracted wound healing, LPS, malignant tumor, innate immunity, alternative therapy.
Case 4: An 86-year-old woman with protracted wound healing became bedridden after cerebral infarction and developed pressure ulcers in the sacral area. The ulcer disappeared in four months with LPS-containing sugar and povidone-iodine application twice a day. There were no adverse effects from LPS application in any of these patients. Conclusion: In diabetes, one cause of protracted wound healing is the low innate immune function, such as the phagocytic activity of macrophages. LPS is expected to promote healing by improving innate immunity, and its beneficial effect of promoting wound healing was clearly demonstrated in the present cases. The topical application of LPS is clinically effective for wound healing and is considered a potentially novel treatment method.

Protracted wound healing, as a postoperative complication, and the delayed healing of pressure ulcers in bedridden patients are often caused by various factors such as malnutrition and decreased local blood flow (1). Various methods are considered to promote wound healing, such as proper nutrition, amino acids for collagen synthesis (2), skin and mucosa graft, and artificial biomaterial dressing (3).

The oral administration of LPS (lipopolysaccharide), which is a glycolipid existing in the extracellular membrane of Gram-negative bacteria, was noted to promote the phagocytic activity of peritoneal macrophages and cure cancer that has metastasized to the lung through Toll-like receptor 4 (TLR4) (4). This may be because of the ability of the LPS to normalize immunity, induce the removal of foreign bodies, anti-inflammatory function, and tissue-repair function (5). Furthermore, in the wound-healing process, TLR4 expression was reportedly induced in keratinocytes, and LPS promoted healing (6). LPS has various structures depending on bacterial strains. For investigating its promoting effects on wound healing in clinical setting, LPS should be used with a unified structure. The wound healing promoting effect of LPS was studied in patients with protracted wound healing by applying LPS-containing 


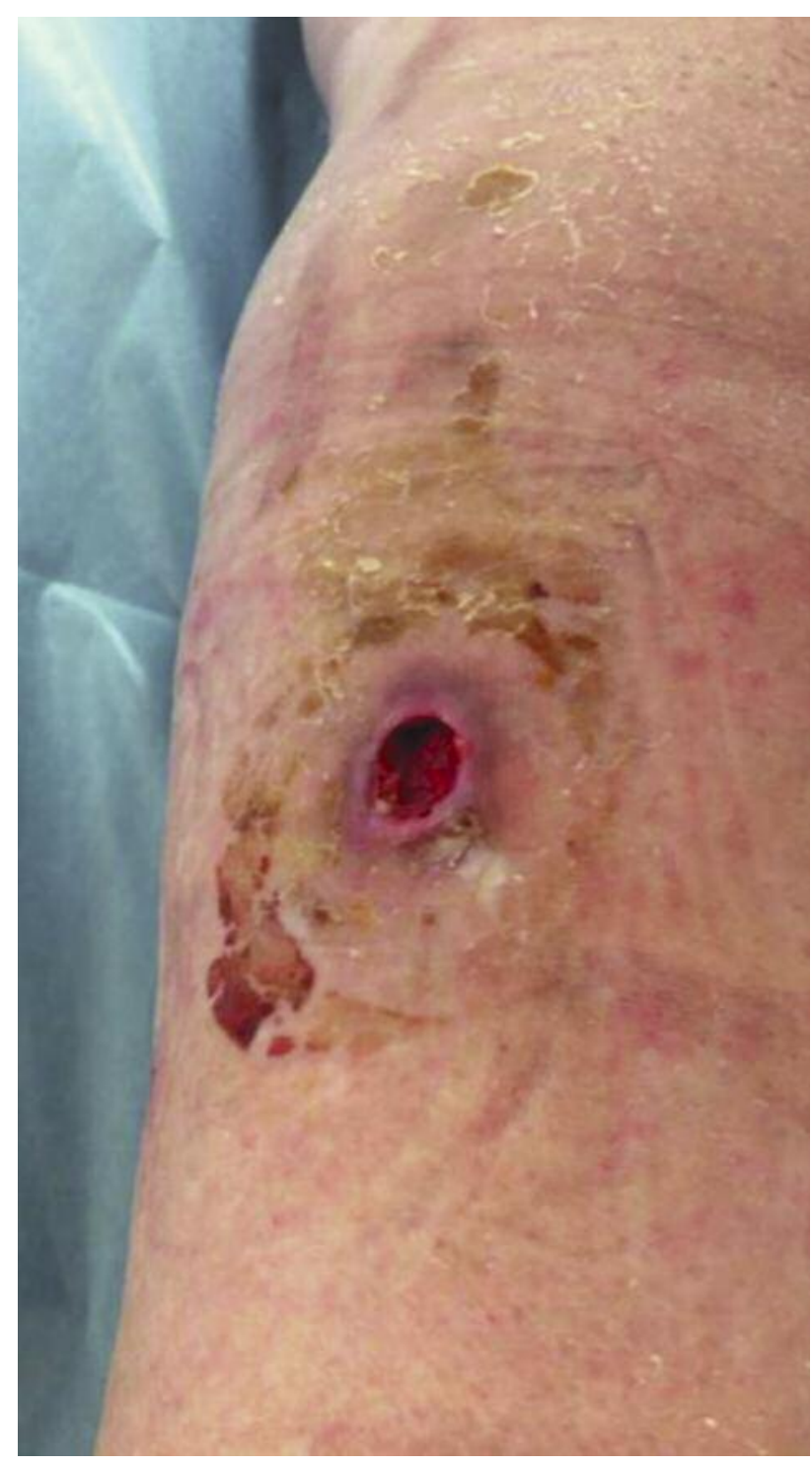

Figure 1. This picture shows protracted wound healing for a month.

ointment derived from Pantoea agglomerans, as we have eating history of it, safety of oral and dermal administration has been confirmed, and its structure is known.

\section{Patients and Methods}

After obtaining written informed consent, the dermal application of undiluted wheat fermentation broth (Institute of Applied Technology for Innate Immunity) in topical cream mixed in a ratio of $3 \%$ ( $3 \mu \mathrm{g} / \mathrm{ml}$ ) was started on four patients who showed protracted wound healing. Wound was evaluated on the basis of the approximate volume change calculated from the width, length, and depth of the wound.

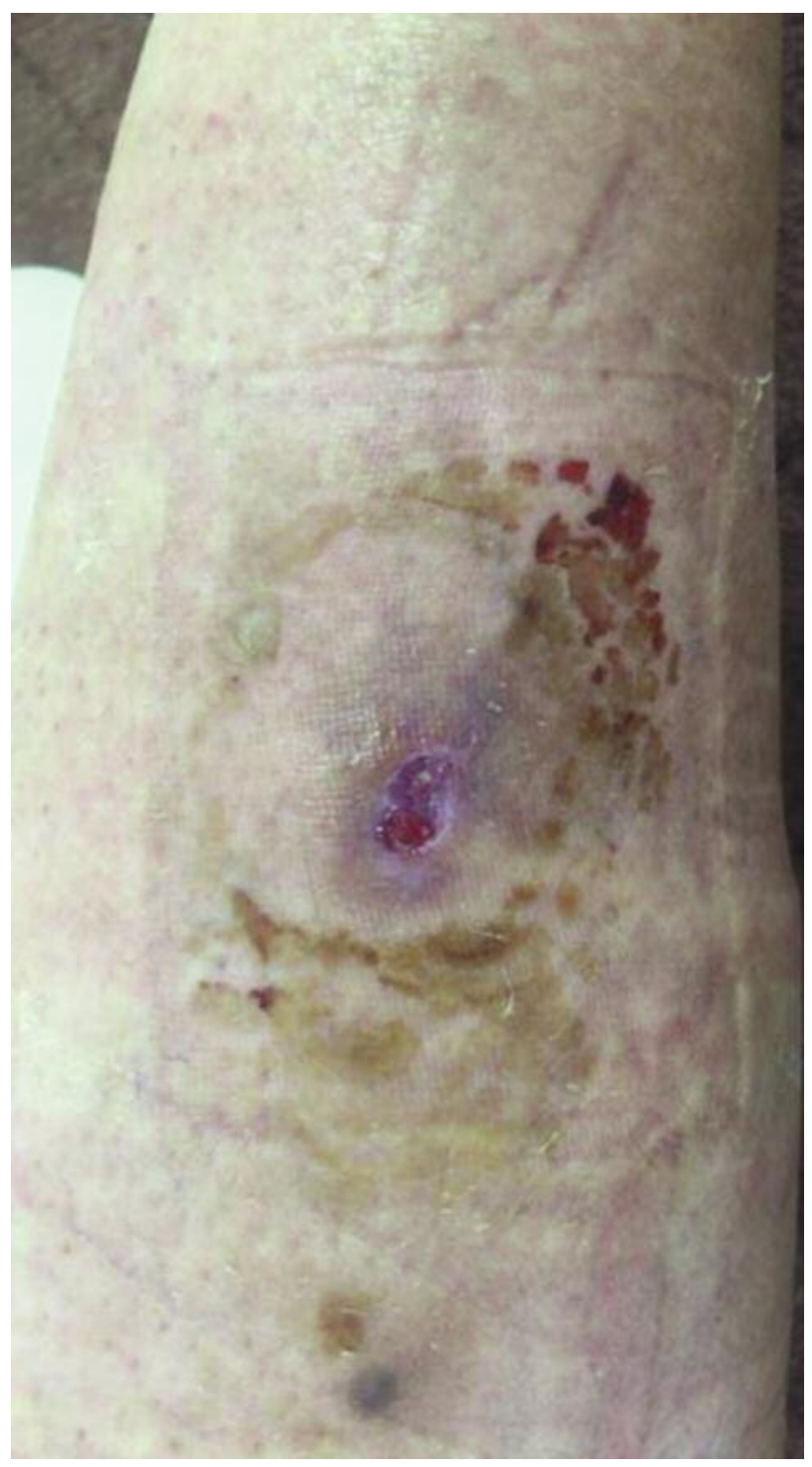

Figure 2. By iodine coating with continuous LPS, the abnormal granulation disappeared and wound healing was performed.

\section{Results}

Case 1. The patient was a 76-year-old man who developed infection at the puncture site $(1.5 \times 2 \mathrm{~cm} \times 0.5 \mathrm{~cm}$, volume $1.5 \mathrm{~cm}^{3}$ ) after the endovascular laser treatment of the right lower extremity varicose veins, which did not show improvement after a month of topical application of gentamicin (Figure 1). This wound condition was considered to be protracted wound healing. Next, the wound was treated with gentamicin application containing LPS and shrank in two weeks $\left(1 \times 1.5 \times 0.5 \mathrm{~cm}\right.$, volume $\left.0.75 \mathrm{~cm}^{3}\right)$. However, abnormal granulation was observed, and the treatment was 


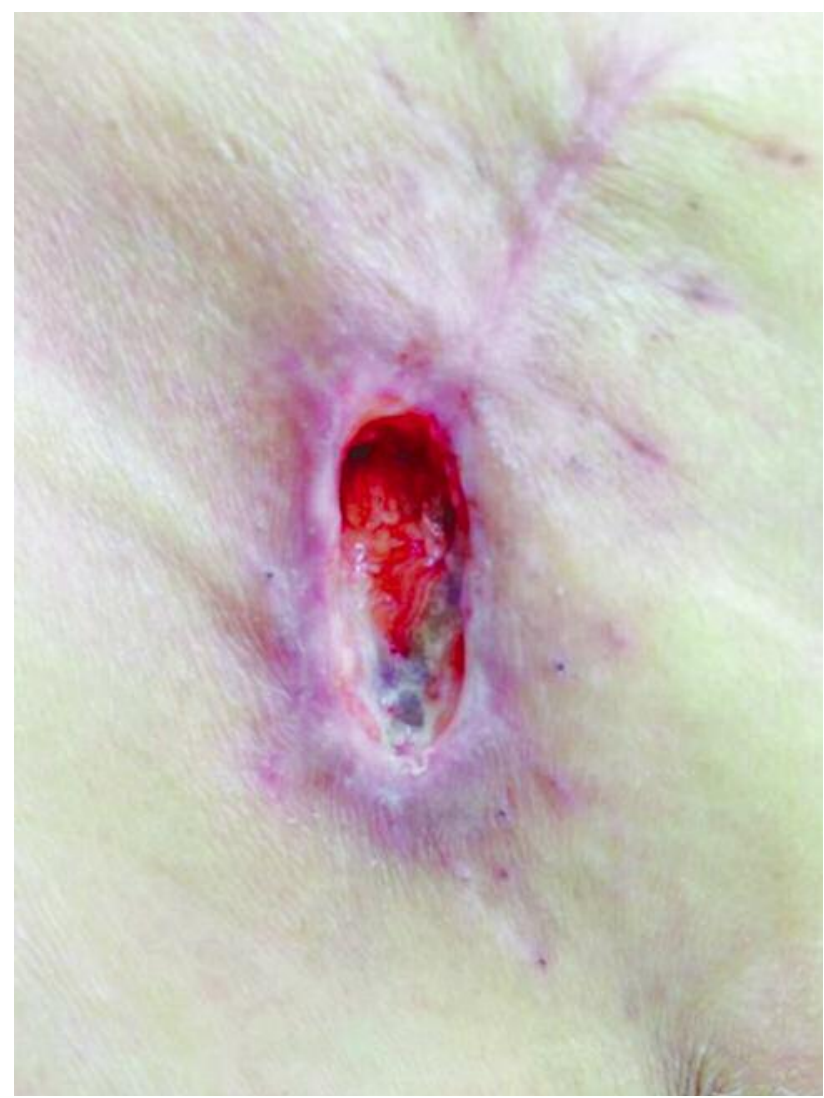

Figure 3. This wound was protracted after a surgical treatment of acute aortic dissection.

switched to iodine coating with continuous LPS application. Subsequently, the abnormal granulation disappeared $\left(0.8 \times 1.2 \times 0.3 \mathrm{~cm}\right.$, volume $\left.0.29 \mathrm{~cm}^{3}\right)$, followed by epithelialization after additional two weeks (Figure 2).

Case 2. The patient was a 72-year-old man who developed a wound infection and had purulent drainage one week after the endovascular laser treatment of the left lower extremity varicose veins. As there was no change observed for a month, the patient was considered to have protracted wound healing. The wound was then irrigated every other day, and gentamicin-containing LPS was applied. At the starting point of this treatment, the wound measured $0.5 \times 1 \times 2 \mathrm{~cm}$, and its volume was $1 \mathrm{~cm}^{3}$. In two weeks, necrotic tissues disappeared and tissue regeneration began, while its depth decreased to $0.5 \mathrm{~cm}$ (volume, $0.25 \mathrm{~cm}^{3}$ ), followed by wound closure after one month.

Case 3. The patient was a 67-year-old woman who developed wound infection in the right inguinal region after a surgical treatment of acute aortic dissection. The wound

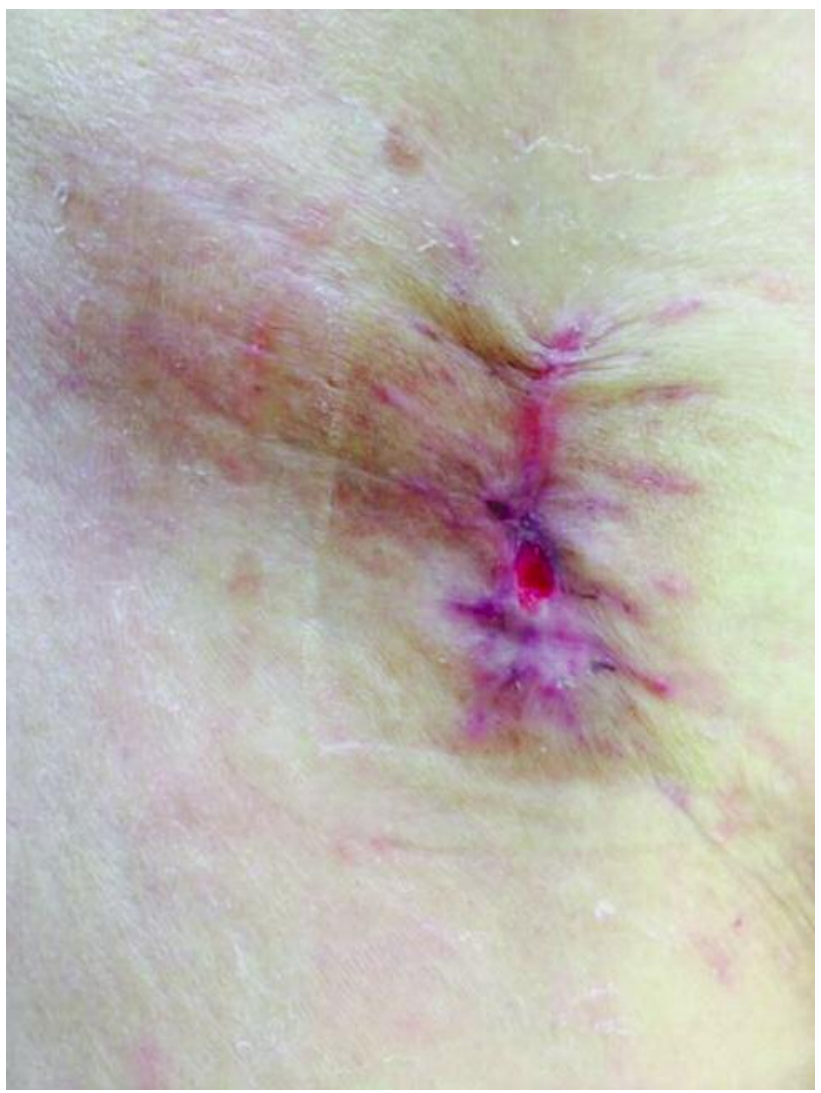

Figure 4. The wound healing was performed by gentamicin application containing LPS.

showed complete dehiscence, and a part of the artificial blood vessel was exposed (about one month after the surgery). It was then considered as protracted wound healing. The wound measured $4 \times 5 \times 2 \mathrm{~cm}$, with a volume of $40 \mathrm{~cm}^{3}$ (Figure 3). For two weeks, wound irrigation was performed every other day, and gauze pack containing LPS was applied. The wound shrank to $1.5 \times 1.5 \times 1 \mathrm{~cm}$, with a volume of approximately $2.25 \mathrm{~cm} 3$. The wound was temporarily sutured. However, a part of the wound dehisced, which shrank to a pinhole size after 10 days of gentamicin application containing LPS. Complete closure was observed in two weeks (Figure 4).

Case 4. The patient was an 86-year-old woman who became bedridden after cerebral infarction and developed pressure ulcers in the sacral area. The wound showed a pocket formation $\left(6 \times 7 \times 2 \mathrm{~cm}\right.$, volume $84 \mathrm{~cm}^{3}$, Figure 5), and wound healing seemed challenging. Therefore, it was considered to be protracted wound healing, and LPS-containing ISODINE $^{\circledR}$ Sugar Paste Ointment (sugar and povidoneiodine) was applied twice daily. The pocket wound 


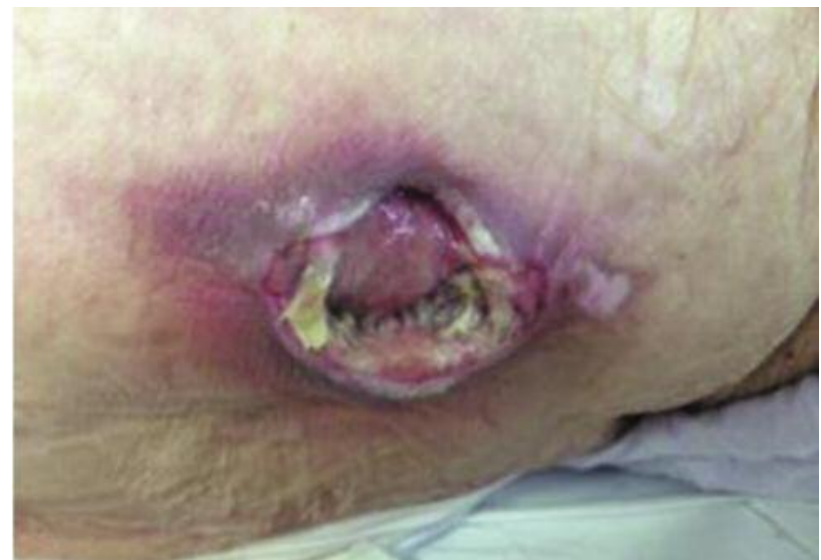

Figure 5. This wound shows pressure ulcers with a pocket formation.

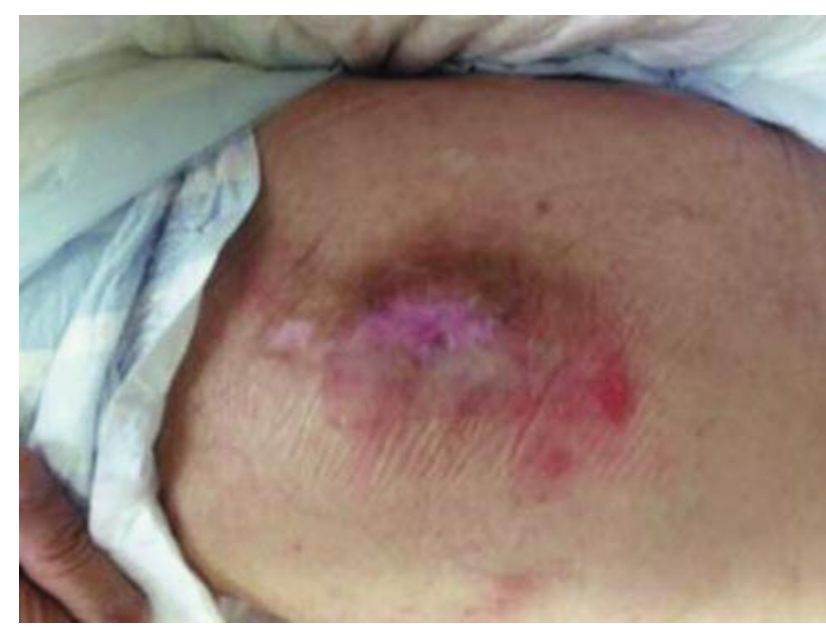

Figure 6. The pressure ulcer disappeared after coating Isodine Sugar Paste Ointment with LPS.

disappeared in two weeks $\left(3 \times 41.5 \mathrm{~cm}\right.$, volume $\left.18 \mathrm{~cm}^{3}\right)$ and the pressure ulcer disappeared after additional two months (Figure 6).

No obvious adverse effects caused by LPS application were reported in any of these cases.

\section{Discussion}

LPS is generally recognized as an inflammatory substance that induces systemic inflammation and allegedly causes endotoxin shock, if injected intravenously. However, the transmucosal administration of LPS reportedly has

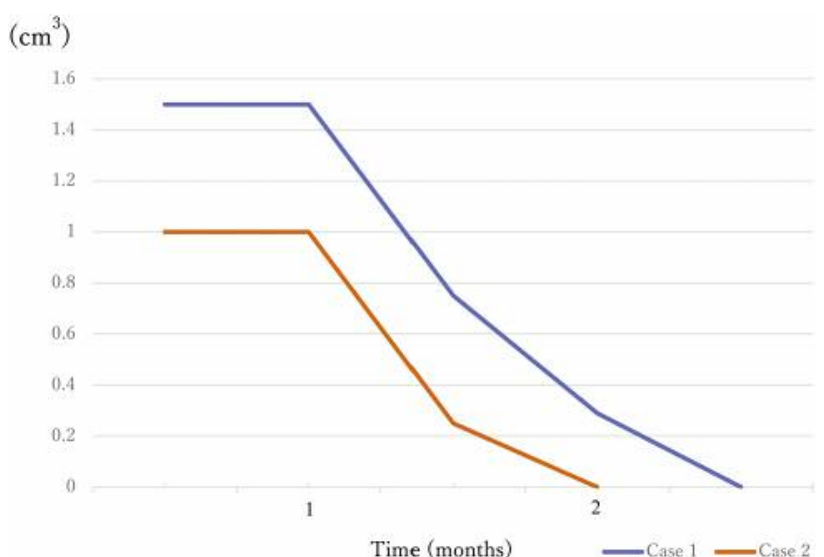

Figure 7. This shows the approximate volume change on Cases 1 and 2 .

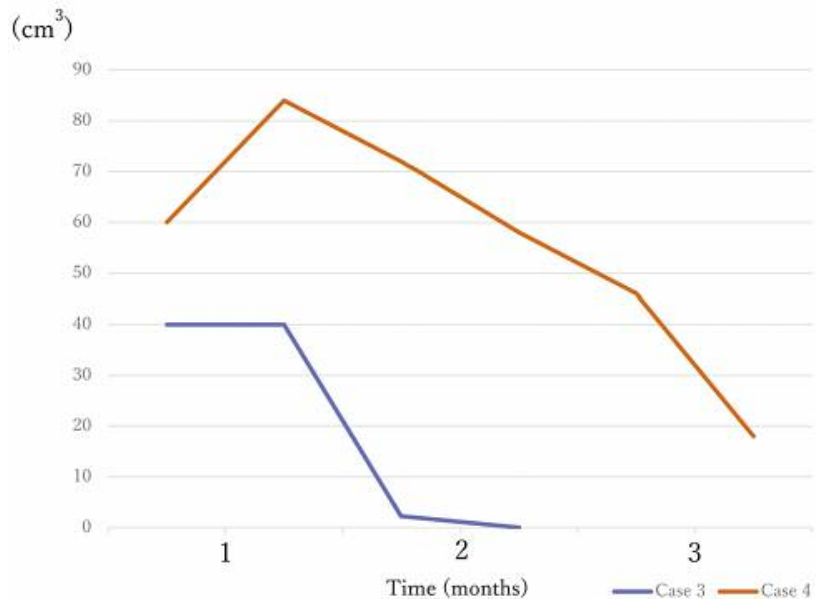

Figure 8. This graph is the approximate volume change on Cases 3 and 4 .

different effects from its inflammatory action. For example, orally administered LPS induces antibacterial peptide synthesis by activating Paneth cells in the small intestine through Toll-like receptor 4 (TLR-4) in the mucous membrane of the small intestine for maintaining immune function (7). Gram-negative bacteria-derived LPS is known to act in favor of repairing the mucous membrane of the respiratory tract and lung diseases if it is present in small quantities (8). In addition, TLR-4 expression is known to be increased in the early phase of the wound healing process, and LPS clearly promotes recovery during the wound healing process. 
During the wound healing process, M1-type macrophages play a role in the early inflammatory phase to remove foreign bodies. M2-type macrophages promote wound healing through suppressing inflammation by IL-10 production, proliferation of fibroblasts by fibroblast growth factor production, and upregulation of angiogenesis by vascular endothelial growth factor production. Meanwhile, macrophages switch from M1-type to M2-type during tissuerepair process. In wounds associated with pathological conditions such as diabetes, the phagocytic activity of macrophages is suppressed, which is considered one of the causes of protracted wound healing.

In the present report, three of four cases were surgical wound infections and one was pressure ulcer. In all these cases, the approximate wound volume did not alter during the initial treatment for approximately one month. However, the approximate volume decreased after the addition of LPS to the treatment and eventually disappeared (Figure 7).

Considering the characteristics of macrophages described above, in all four cases, macrophages seemed to have remained M1 phenotype, but LPS addition increased M2 type macrophages to promote wound healing.

Cases 1 and 2 included wound infection from percutaneous surgeries, and outpatient care was sufficient for treating the wounds. Moreover, case 3 included wound infection after a surgical treatment for aortic dissection. A part of the artificial blood vessel that was used in the surgery was exposed, and inpatient hospital care was necessary. However, the patient strongly refused hospitalization; therefore, we added LPS to the ointment in addition to wound irrigation, which can be performed under outpatient care.

Case 4 included a pressure ulcer with a large pocket formation, which usually indicates a skin graft surgery. However, the patient was eating well and had a good nutritional status, which enhanced wound healing, and in turn enhanced the effect of LPS.

\section{Conclusion}

The topical administration of the appropriate amount of fermented wheat-derived LPS is clinically effective and is likely to become a novel method of wound treatment.

\section{References}

1 Terashima $\mathbf{M}$ and Tanizawa $\mathrm{Y}$ : Anastomac and Intra-abdominal abscess (Upper gastrointestinal tract). Surg Treat 102(5): 739746, 2010.

2 Saito Y, Mimatsu K and Kawasaki A: A case of improvement of wound healing associated with surgical site infection by oral administration of the specialized amino acid supplement consisting of CaHMB, L-arginine and L-glutamin (e Abound ${ }^{\mathrm{TM}}$ ) Parenteral and Enteral Nutrition 27: 81-85, 2012.

3 Kaneda T, Chujyo A and Torii S: The clinical use of LPS (Lyophilized porcine skin) as a temporary biological dressing in oral surgery. J Oral Maxillofac Surg 27: 132-140, 1981.

4 Atsutomo Morishima and Hiroyuki Inagawa: Clinical effects of orally administered lipopolysaccharide derived from Pantoea agglomerans on malignant tumors. Anticancer Res 36: 3747-3752, 2016.

5 Inagawa $\mathrm{H}$, Kohchi $\mathrm{C}$ and Soma $\mathrm{G}$ : Usefulness of oral administration of lipopolysaccharide for disease prevention through the induction of priming in macrophages. Anticancer Res 34: 4497-4501, 2014.

6 Chen L, Guo S, Ranzer MJ and DiPietro LA: Toll-Like Receptor 4 has an essential role in early skin wound healing. J Invest Dermatol 133: 258-267, 2013.

7 Koff JL, Shao MX, Kim S, Ueki IF and Nadel JA: Pseudomonas lipopolysaccharide accelerates wound repair via activation of a novel epithelial cell signaling cascade. J Immunol 177: 86938700, 2006.

8 Boniakowski AE, Kimball AS, Jacobs BN, Kunkel SL and Gallagher KA: Macrophage-mediated inflammation in normal and diabetic wound healing. J Immunol 199: 17-24, 2007.

Received April 11, 2018

Revised June 5, 2018

Accepted June 11, 2018 